\title{
Crisis en la ciencia y en la espinitualidad: i un fenómeno contemporáneo? Aproximación desde los planteamientos de Husserl, Prigogine y desde la tradición taoísta
}

\author{
Ana Cecilia Vallejo Clavijo*, José Arlés Gómez Arévalo**
}

Recibido: 15 de marzo de 2010 Revisado: 28 de mayo de 2010 Aprobado: 6 de julio de 2010

\section{Resumen}

El tema de la ciencia presente en la modernidad, en particular, de la física newtoniana y galileana, y sus influencias en la filosofía de Descartes, ha sido retomado de forma crítica tanto desde los planteamientos filosóficos fenomenológicos de Edmund Husserl como desde las ciencias de la complejidad estudiadas por Ilya Prigogine. Ambas posturas, la filosófica y la científica, destacan las limitaciones del pensamiento moderno proponiendo una revisión y un aporte desde lo humano y desde las ciencias mismas. Por otra parte, desde la visión de algunas de las culturas del lejano Oriente: China, India y Japón, se percibe la complejidad de las relaciones entre el hombre y la naturaleza, a la vez que se pretende establecer un diálogo con la manera como se ha abordado la problemática en el mundo occidental, sus posibles retos y aportes frente a un mundo cada vez más tecnocratizado y globalizado, signado por las desigualdades socio-económicas, las paradojas y contradicciones frente a sí mismo, a los demás y al universo.

\section{Palabras claves}

Crisis, epojé, termodinámica, cosmos, unidad, diversidad, espiritualidad.

* Docente del Departamento de Humanidades. Licenciada en Filosofía y Humanismo de la Universidad Santo Tomás. Estudios de Maestría y Docencia Universitaria en la Universidad Santo Tomás y Doctorado Filosofía Pura en la Pontificia Universidad J averiana. Co-investigadora del grupo de Investigación Ciencia-Espiritualidad, reconocido por Colciencias. Correo electrónico: anacelv@ hotmail.com

** Docente del Doctorado en Educación de la Universidad Santo Tomás (USTA). Doctor en Teología (Pontificia Universidad Urbaniana, Roma). Magíster en Filosofía Latinoamericana (USTA, Bogotá). Licenciado en Teología (Pontificia Universidad Javeriana, Bogotá). Especialista en Orientación Sexual (Universidad Manuela Beltrán, Bogotá) y Especialista en Educación Filosofía Colombiana (USTA, Bogotá). Director de la Línea Ciencia-Espiritualidad. Correo electrónico: angel777abc@ hotmail.com 


\title{
Crisis in science and spirituality: a contemporary phenomenon? Approach from the approaches of Husserl, Prigogine and from the taoist tradition
}

\author{
Ana Cecilia Vallejo Clavijo, José Arlés Gómez Arévalo
}

\begin{abstract}
The theme of science presented in modernity particularity in Galilean and Newtonian physics and its influence on the philosophy of Descartes, have both been critically revisited by the phenomenological philosophical approaches from Edmond Husserl as well as by the sciences of complexity from Ilya Prigogine. Both positions, the philosophical and scientific ones highlight the limitations of modern thought by proposing a review and input from human and from the sciences themselves. Moreover, from the perspective of some of the cultures of the Far East: China, India and Japan, complexity of the relationship between man and nature is perceived, while we intend to establish a dialogue with the way they addressed the problem in the western world, its potential challenges and contributions in an increasingly global and technical world pointed by socio-economic disparities, paradoxes and contradictions facing yourself, others and the universe.
\end{abstract}

\section{Key words}

Crisis, epojé, thermodynamics, cosmos, unity, diversity and spirituality.
Recibido: 15 de marzo de 2010 Revisado: 28 de mayo de 2010 Aprobado: 6 de julio de 2010 


\section{INTRODUCCIÓN}

En la primera parte de este trabajo investigativo se hará referencia al libro de E. Husserl (2008), La crisis de las ciencias europeas y la fenomenología trascendental, en el que se plantean los siguientes interrogantes: ¿Hay efectivamente crisis en las ciencias, habida cuenta de sus éxitos incesantes?, ¿se ha vuelto cuestionable el auténtico carácter científico?..., preguntas que apuntan a las pretensiones de la psicología como ciencia positiva. Esta crítica emprendida por Husserl no se refiere, como él mismo lo anota, a la legitimidad de la metodología científica, sino más bien, a la visión total del mundo por parte de los seres humanos que, durante la segunda mitad del siglo XIX, se dejaron determinar y cegar: "por las ciencias positivas y por la 'prosperity' de que son deudores, significó un alejamiento de las preguntas decisivas para una auténtica humanidad" (Husserl, 2008, p. 49-50). Refiriéndose al positivismo, destaca cómo las "meras ciencias de hechos, hacen meros seres humanos de hechos". Así, la ciencia de los cuerpos no tiene manifiestamente nada que decir, puesto que se abstrae de ellas todo lo subjetivo. La pregunta que surgiría entonces es si se puede estudiar el ser humano desde la objetividad, la legalidad y la comprobación propias del espíritu positivista. Para Husserl, las preguntas referidas sobre lo humano se convierten en "supremas y últimas" y apuntan al sentido de la razón. Estas preguntas supremas y últimas hacen referencia al sentido o al sinsentido de la existencia humana, la razón y la sinrazón sobre los seres humanos portadores de humanidad. Realzando la importancia de la reflexión filosófica, Husserl considera que el destino de la filosofía será entonces buscar el camino del "autodesvelamiento".
Asimismo, se plantea una crítica hacia las ciencias y la filosofía de la modernidad, desde el método de la complejidad en Edgar Morin y de las ciencias de la complejidad desde Prigogine, crítica que va dirigida primeramente hacia la lógica tradicional, en el intento por formular un nuevo tipo de racionalidad que incide sobre el método. Se rescata, además, el importante significado que para Prigogine tiene la flecha del tiempo en la dinámica de las trasformaciones complejas relacionadas con el ser vivo. El estudio de las ciencias de la complejidad es una de las formas generales utilizada para designar aquel conjunto de fenómenos, comportamientos y sistemas que no cabe explicar apelando a los criterios formales presentes en la ciencia de la modernidad del siglo XIX, en la que se impone estrictamente la aplicación de una lógica formal que sustenta la idea de causalidad lineal y de un pensar analítico.

Estas ciencias de la complejidad, como se dijo anteriormente, son portadoras de una nueva de racionalidad, haciéndose necesaria la demanda de otro tipo de lógica que haga justicia a la complejidad de lo real y se exprese de forma diferente a la adoptada por la lógica lineal, en la que se estudia la cosa en sí misma, sin tener en consideración el juego de relaciones que la rodea. En esta nueva lógica propuesta, tiene cabida la diferencia o el reconocimiento de la no identidad en su autonomía y singularidad, es decir, lo otro o el otro. La inclusión de lo otro, o el otro, lleva a adoptar una posición epistemológica de complementariedad-reciprocidad. Esta nueva lógica fue propuesta y aplicada por los físicos cuánticos Bohr y Heinsenberg, al estudiar el componente onda-partícula presente en la naturaleza. 
En esta nueva forma de razonar, se prolonga la diferencia de forma dialógica y se intenta confrontar la identidad con la diferencia, incluyéndola en un proceso dinámico, para dar como resultado una nueva identidad: la síntesis, que está configurada como algo más que la suma de las partes y es ahí cuando la realidad denominada "emergencia creadora" cobra importancia para las ciencias de la complejidad.

En la segunda parte del presente artículo, desde el cuestionamiento del llamado "desarrollo humano", en la época actual, que se ha distinguido por un constante incremento de la insolvencia racional del hombre y de su poder socio-económico para actuar con sabiduría y conciencia frente a la naturaleza y al cosmos, se analiza la relación que se estableció en las antiguas tradiciones de Oriente con respecto a la naturaleza. En dicha relación, podemos encontrar algunas claves para reorientar la posición del hombre contemporáneo frente a la naturaleza, en términos de unidad y de equilibrio correlacional.

I Parte

Crisis en la Ciencia y

EN LA ESPIRITUALIDAD:

PLANTEAMIENTOS

FENOMENOLÓGICOS DE EDMOND

HuSSERL Y DESDE LAS CIENCIAS

DE LA COMPLEJIDAD EN ILYA

Prigogine

\section{El papel de la filosofía y la reflexión en la ciencia según Husserl}

Husserl, en su Crítica a la ciencia moderna occidental, plantea la necesidad de restablecer la filosofía teórica de observación reflexiva, crítica, autónoma y libre, con total ausencia de los prejuicios impuestos por la ciencia, seguida por una autonomía práctica. En su propósito de concederle a la filosofía la importancia que se merece, considera que ésta fue entendida desde los primeros siglos de la humanidad como una ciencia "omniabarcadora", "la ciencia de la totalidad de lo que es"; por tal razón, la filosofía se considera una ciencia objetiva universal. En este estudio, se hace patente una paradoja que tiene que ver con la dinámica de la humanidad, a saber: si el ser humano deviene de un problema metafísico, entonces él mismo está en cuestión como ser racional y si se cuestiona su historia, igualmente se está cuestionando su sentido; ello implicaría la urgencia de trabajar el problema de la razón de la historia. Por otra parte, y desde la filosofía, se encuentra que los problemas de la razón misma y lo existente se vuelven cada vez más enigmáticos; es así que el problema universal de la relación entre la razón y lo existente en general, "el enigma de todos 
los enigmas", se convierte en el auténtico tema. Para Husserl, la filosofía considerada históricamente es el intento por lograr, en mayor o menor medida, la idea conductiva de la humanidad y la historia: “deja de ser de la humanidad finita para llegar-a-ser una humanidad de tareas infinitas" (Husserl, 2008, p. 50). A pesar de que el hombre en su actitud natural vive perdido y alienado en el mundo, sin embargo, puede tomar distancia frente a él y asumir una actitud reflexiva; se trata de que el hombre desarrolle la posibilidad concreta para decidir libremente y tomar conciencia de un presente viviente.

\section{ACerca del método}

Husserl encuentra que la psicología, en la búsqueda por el método científico que se adecúe al problema del mundo de la vida, no ha sido capaz de preservar el problema del ser del mundo, de la vida; por tal razón, propone una epojé referida al conocimiento que se lleva a cabo en las ciencias objetivas. $\mathrm{Su}$ función es asegurar el cambio y la transición que apuntan a las experiencias del mundo de la vida; parte de una reflexión trascendental fenomenológica sobre ese mundo y las formas de darse que

apunta a estructuras ontológicas y fenomenológicas a priori y experiencias del mundo de la vida, se incluye en esto la una actitud natural que reflexiona sobre los diversos modos en que se da el mundo de la vida, surgiendo la conciencia unitaria simple "ser" del mundo (Husserl, 2008, p. 149).

El camino que se transita y que parte de la actitud natural a la actitud filosófica lleva implícito una paradoja, que consiste precisamente en advertir cómo el ser sujeto para el mundo es también objeto de este mundo; paradoja que se resuelve por medio de la diferenciación entre: "la subjetividad objetiva como parte del mundo y subjetividad trascendental que es constitutiva del mundo" (Husserl, 2008, p. 35).

Además, con la epojé trascendental no se pierde el mundo en el momento en que se lleva a la práctica. Ello significa que también el filósofo, en la epojé, debe "vivir naturalmente" la vida natural. La reducción trascendental viene a ser una superación de las limitaciones propias del conocimiento natural y objetivo, que en cierto momento puede manifestarse unilateral, abstracto, superficial o vulgar. Con la reducción fenomenológica se abre paso una mayor comprensión de nosotros mismos como humanidad: "en nuestro filosofar somos funcionarios de la humanidad". Husserl (2008) nos hace ver cómo las verdaderas luchas son las de la filosofía, las luchas espirituales que se orientan por el sentido de una auténtica humanidad y el camino para poner en marcha una filosofía universal; así se expresa:

Si el ser humano es un ser racional sólo lo es en la medida en que su total humanidad sea humanidad racional orientada hacia la razón de un modo latente [...] la Filosofía y la Ciencia serían: el movimiento histórico de la manifestación de la razón universal, como tal, congénita para la humanidad. Movimiento aún no terminado en donde la razón plenamente consciente de sí manifiesta de forma propia una filosofía universal progresiva que se autorregula según un método apodíctico (p. 59). 


\section{LA INTUICIÓN CREADORA EN HUSSERL: LA CORRELACIÓN HOMBRE-MUNDO}

En su Crítica..., Husserl nos da a conocer su "intuición creadora", que consistió en intuir la existencia de una correlación entre el hombre y el mundo, tarea altamente difícil dado que la experiencia humana no deja racionalizar, explicitar ni ser entendida plenamente:

Captar el significado de la correlación hombre-mundo no es tarea fácil debido a que la "correlación" se revela a veces como mundo vivido ocultando la vida que vive este mundo, otras veces se revela como la vida que se experimenta este mundo ocultando todo el sentido del mundo vivido (Herrera, 2002, p. 42).

El mundo al que se refiere Husserl es el mundo de las "cosas" de los cuerpos percibidos, que se da por presupuesto; es el mundo familiar: "siendo para nosotros de antemano: 'suelo para todos' se trate de la práctica teórica o estrateórica" (Iribarne, 2002, p. 38). Desde el punto de vista epistemológico en esta correlación mencionada, además de que el objeto de la conciencia tiene presencia gracias a los modos de darse en ella misma, el sujeto es descrito desde una doble dimensión unitaria, es decir, es a la vez sensibilidad y razón, totalidad estructurada que experimenta el mundo de forma significativa.

Por otra parte, el hombre viene a ser una estructura dotada de movimiento que "experimenta el mundo" intencionalmente, referido a ese mundo exterior y en el yo que es portador de actos; es además totalidad de vivencias intencionales motivadas por su ser histórico y libre. Sólo gracias a mi estructura temporal que retiene el pasado y anticipa el futuro, puedo experimentar el mundo y experimentarme a mí mismo. Lo anterior nos lleva a afirmar que toda experiencia posee una estructura de horizonte y sin la temporalización de mi yo la reflexión sería imposible. Este horizonte hace referencia precisamente a la totalidad de lo perceptivo o perceptible de los fenómenos en cada uno de los actos cognitivos particulares y cargados de sentido, el horizonte de temporalidad abierto despierta otros horizontes no del todo comprendido:

La amplitud y la profundidad de conjunto de este sentido, en su totalidad infinita, alcanzan dimensiones axiales tan pronto se llega a dominar en cierta medida la configuración del sentido, se abren entonces los problemas de la totalidad como los de la razón universal (Husserl, 2008, p. 211).

\section{EL MUNDO DE LA VIDA (LEBENSWELT) Y EL MUNDO DE LA CIENCIA}

El mundo de la vida es el mundo "predado", el "a-priori concreto", con una existencia anterior a la ciencia. Este mundo continúa su modo de ser en la época de las ciencias y se constituye como fundamento de ellas. En él, a través de las experiencias, tienen lugar las donaciones originarias de sentido. Husserl se refiere al mundo de las funciones cinestésicas que se manifiestan corporalmente:

En todas las verificaciones de la vida natural de intereses que se sostienen puramente en el mundo de la vida juega un papel prominente la vuelta a la intuición "sensiblemente experienciante" [...] Toda cinestesia, todo "yo me muevo", "yo hago" están vinculados entre sí en la unidad universal, siendo 
el reposo cinestésico un modo del "yo hago" (Husserl, 2008, p. 148).

Para comprender mejor la contraposición entre el mundo de la vida y el mundo de la ciencia, podemos remitirnos a las relaciones que se establecen entre el mundo de la vida y el mundo especializado y cómo se constituye este último. El mundo de la ciencia o especializado está constituido a partir de una idea finalizante y tiene como pretensión restringir su teoría a un "horizonte interesado" o a una ciencia particular; consecuencia de ello es que su finalidad se torna igualmente particular. Por el contrario, el mundo de la vida posee un sentido más universal e implícitamente se interrelaciona con los mundos derivados. Sustituir o intercambiar el mundo de la vida por el mundo particular del científico significa tomar la parte por el todo y reducir el concepto de realidad. Bajo estas condiciones, Husserl considera que la ciencia positiva no se halla en condiciones de asumir al ser humano como problema metafísico, ni se ocupa de la marcha de la razón en la historia; en otras palabras, la ciencia positiva perdió su fe en la filosofía universal, en su ideal y en el alcance de su método.

En su crítica a la ciencia de la modernidad en Occidente se refiere puntualmente a dos figuras claves en la filosofía y en la ciencia: Descartes y Galileo, mostrando cómo la influencia de Galileo en Descartes se evidencia en la aceptación del racionalismo objetivista que se vio claramente presente en la filosofía moderna. Critica, por otra parte, la posición dubitativa cartesiana y asume que no se puede dudar del mundo; esta posición se torna imposible dado que su certeza se impone como condición de toda praxis, ya sea ésta científica o extracientífica. El error de Descartes, según esta crítica, radicó en aceptar acríticamente el mundo de la física galileana, mundo cerrado de cosas, encapsulado y vacío. Iribarde (2008) hace referencia a la crítica que realiza Husserl sobre Galileo en su intención de matematizar y objetivizar la naturaleza:

Todo lo que se anuncia como real en las cualidades sensibles específicas, debió tener su índice matemático en el acontecimiento de la esfera de las formas [...] y que por este medio debiera ser posible (aunque indirectamente y por un método inductivo particular ex datis todos los acontecimientos del lado de los contenidos y con eso determinarlos objetivamente (Husserl, 2008, p. 17).

Husserl considera que con la matemática de Galileo, desde el punto de vista del conocimiento, se pretende superar el aspecto "meramente subjetivo" y la relatividad del mundo sensible precientífico, para dar paso al mundo de las ideas objetivas, las llamadas "cualidades primarias". Mediante la matemática pura y el arte práctico de la medida se intenta llegar a una posible previsión inductiva y certeza empírica. Desde esta lógica, se espera que el mundo intuido de la vida real-efectiva resulte de la matematización y sus fórmulas. Para Husserl, este exceso de "aritmetización" y de tecnificación ocasionó un vaciamiento de sentido de la ciencia matemática de la naturaleza. Sobre este tema Husserl (2008) expresa:

En la secuencia del esfuerzo por despertar un conocimiento "filosófico" determinante del ser del mundo "verdadero", objetivo "el arte de la medida empírica y su función objetivizante al modo empírico-practico, bajo la transformación del interés práctico en interés puramente teórico el conocimiento "filosófico" fue idealizado y así se convirtió en el modo de pensar puramen- 
te geométrico. El arte de la medida se convierte en precursor de la geometría finalmente universal y su "mundo" de puras formas-límite (p. 70).

\section{LAS CIENCIAS DE LA COMPLEJIDAD}

\section{Acerca del método}

El problema del método desde la perspectiva de la complejidad ha sido ampliamente trabajado por Edgar Morin en su libro El Método. En este estudio, se plantea la necesidad de sustituir el paradigma de la disyunción/reducción/unidimensialización, por el de la distinción/conjunción, permitiendo distinguir sin desarticular, asociar sin identificar o reducir. Desde esta perspectiva, no pierden legitimidad los frutos conseguidos por la ciencia; gracias a ella, se han adquirido conocimientos sin precedentes en el mundo físico, biológico, sociológico, psicológico, etc.; sin embargo, desde el método de la complejidad se insta a que cuestionemos nuestro conocimiento de forma reflexiva con el fin de encontrar grados de error, de ignorancia y de ceguera que pueden estar presentes.

Esta reflexión va dirigida no a la ignorancia humana en general, sino a la disimulada, agazapada, la que se instala en el hombre poseedor del conocimiento científico, amenazado progresivamente por el error, la ignorancia, la ceguera. Desde esta postura, se insta a adoptar un nuevo método, adoptar una nueva forma de organización de nuestro conocimiento, que implicaría la adopción de un nuevo paradigma epistemológico. Desde Morin se debe transformar el descubrimiento de la complejidad en el método de la complejidad. Desde esta nueva postura, la relación de causalidad lineal que se presenta en los fenómenos estudiados no se manifiesta tan claramente; por el contrario, aparecen varios niveles de causalidad, generándose interacciones. Estos niveles de causalidad serían:

Primero: en el que toda causa produce tales efectos (causalidad lineal), segundo: en el que el efecto puede retroactuar o hacer disminuir las causas (causalidad retroactiva), tercero: en el que los efectos y las causas son necesarios para el proceso que los genera (causalidad recursiva)... (Morin, 1990, p. 123).

\section{Crítica de la teoría de la complejidad a la física moderna}

Para Prigogine, el estudio de las ciencias de la complejidad se denomina sistemas dinámicos no lineales alejados del equilibrio o sistemas adaptativos. Dichos sistemas se caracterizan por presentar una realidad que obedece a fluctuaciones, inestabilidades, procesos autorganizativos, azar, caos, orden, desorden, bifurcaciones; en este sentido, la complejidad y la no linealidad se implican mutuamente. Los nuevos conceptos o categorías mencionadas, que surgen en el contexto de las ciencias complejas, pretenden dar nuevos aportes y romper con las limitaciones existentes en la ciencia moderna newtoniana.

La crítica emprendida hacia la ciencia moderna desde las ciencias de la complejidad auguran una metamorfosis en las ciencias. En esta crítica se muestra cómo el postulado determinista, que depende de forma categórica de la experimentación y se orienta fundamentalmente hacia la técnica, se torna cada vez más indiferente frente al mundo de la vida humana y la sociedad en general. 
Esta crítica va dirigida, además, y de forma especial como se expresó anteriormente, a la física clásica de corte positivista que pretendió constituirse como único conocimiento posible, descartando la validez de otros saberes posibles.

Además, desde esta crítica, se encuentra que la lógica presente en la ciencia moderna es necesariamente formal y en su afán por destacar la legalidad universal y el determinismo, no tiene en cuenta el problema de la medición de las escalas (para la física de la modernidad: en cada instante, todo viene dado por el dato inicial con absoluta necesidad y predictibilidad), lo que equivale a afirmar que no existe diferencia alguna entre pasado y futuro (tiempo reversible). Lo anterior trae como consecuencia el desconocimiento de singularidades, sobresaltos, accidentes e incertidumbres. Desde la física moderna (que trabaja el movimiento de los planetas o el péndulo ideal), existe un impedimento para plantear el tiempo asociado a la evolución biológica o el de las sociedades.

La nueva posición epistemológica planteada desde las ciencias de la complejidad transformará radicalmente las relaciones del hombre con la naturaleza, los otros seres humanos y la sociedad en general. Desde Prigogine, la ciencia newtoniana no dialoga con la naturaleza, sino que impone sus reglas fijas. Además, el científico no solo no conoce la cosa en sí kantiana, sino que también desconoce o no aborda los verdaderos problemas de la humanidad.

Ahondando en el tema del tiempo, se encuentra que uno de los temas de estudio presentes en la tradición occidental ha estado referido a la temporalidad, el devenir, la permanencia y el cambio. En el modelo de la ciencia moderna, en particular de la física, el tiempo ha estado referido a la regularidad y periodicidad. Esta concepción de la dinámica hunde sus raíces en la física de Aristóteles. En el estudio sobre la física aristotélica, se encuentran planteamientos acerca del movimiento constante, regular y eterno de los astros celestes, ajeno a un primer principio creador. En la modernidad, esta dinámica ordenada y regular presente en el universo conlleva pensar en ello como si fuese una gran máquina, un sistema cerrado regido por leyes inmutables, predecibles y de gran precisión:

Las leyes de la naturaleza enunciadas por la física representan por tanto un conocimiento ideal que alcanza certidumbre. La naturaleza es una autómata que podemos controlar, por lo menos en principio. La novedad, la elección, la actividad espontánea son solo apariencias relativas al punto humano (Prigogine, 1997, p. 19).

Por otra parte, desde las ciencias de la complejidad, se muestra cómo la termodinámica, asociada a la ley de la entropía, conducía inexorablemente al equilibrio (sistemas cerrados), a diferencia de la dinámica de los procesos irreversibles (sistemas abiertos), en los que el tiempo desempeña un papel fundamental y constructivo en la realidad, lo cual generó nuevas realidades y formas de conocimiento.

Los procesos dinámicos irreversibles estudiados por Prigogine conllevan estructuras disipativas y sistemas alejados del equilibrio. En estos sistemas se reconocen la existencia de inestabilidades, de atractores extraños, galaxias que nacen y mueren. Los sistemas abiertos o lejos del equilibrio son altamente sensibles a novedades generando dinámicas no lineales. Así, un 
fenómeno como es la evolución comporta procesos de irreversibilidad y presentan irrupciones, discontinuidades, quiebres, disrupciones y formas de organización espontánea, es decir, que conducen a procesos autoorganizativos.

De la misma manera, se admite que en nuestro mundo cotidiano existen dinámicas fluctuantes en la economía y en los mercados financieros en la que se constatan la presencia de factores inciertos. Dado que la emergencia de realidades más complejas no son objeto de estricto control ni predicción, ello lleva a reconocer una nueva modalidad del conocimiento, una nueva etapa, que según Prigogine conduce al "fin a las certidumbres". Se podría establecer de manera general que: la ganancia que ha tenido el estudio de los sistemas complejos consiste en establecer atención a las transformaciones inesperadas e inciertas y donde el factor prioritario no es el control.

Es importante resaltar que la extensión de la dinámica a los sistemas inestables y caóticos hace posible superar la contradicción entre las leyes reversibles de la dinámica y la descripción evolucionada asociada a la entropía. Esta descripción se remite a los seres vivos con capacidad de evolucionar. Por otra parte, la aceptación del componente temporal facilitaría la presencia de temas fronterizos $\mathrm{u}$ horizontes de convergencia que servirían de puente entre las ciencias sociales, humanas y naturales. Desde esta perspectiva, hay un reconocimiento de que vivimos en un mundo abierto y la historia misma es el resultante de la conjugación entre determinismo y azar. Desde las ciencias de la complejidad, asumir el estudio de la naturaleza únicamente a través de la abstracción derivada de los cuerpos en movimiento impide otorgar un sentido a la noción de creatividad que viene a ser el principio de la novedad. Prigogine (1997) considera que el tratar de entender la naturaleza no es lo mismo que controlarla:

Uno de los grandes proyectos del pensamiento occidental ha sido entender la naturaleza. No debe confundirse con la idea de controlar la naturaleza. Ciego sería el amo que creyera entender a sus esclavos porque le obedecen ( $p$. 173).

\section{Ciencia y filosofía}

El legado de la modernidad, sumado a la fragmentación y especialización del conocimiento traducido en la formación de las disciplinas, delimitará la constitución de dos culturas que parecen excluirse $u$ oponerse: una, que tiene que ver con la investigación científica de corte positivista, y la otra, con la reflexión filosófica. Esta distinción se traduce en la ya conocida expresión entre las ciencias duras y las ciencias blandas. Frente a esto y desde el método de la complejidad, se plantea la posibilidad de una nueva organización del conocimiento, en la que se vislumbre un posible encuentro o acercamiento y puedan converger o confluir lo simple y lo complejo.

Desde esta perspectiva se insiste en que la ciencia por sí misma no puede cerrar sus fronteras desconociendo o descartando otros enfoques y aportes metodológicos dados por las ciencias humanas, la filosofía y el arte. Es necesaria una nueva comprensión de las ciencias, por ello, se insta a iniciar una relectura sobre las relaciones siempre cambiantes entre el hombre y la naturaleza. El resultado de este encuentro implicaría la transformación y creación de nuevos len- 
guajes, conceptos, categorías y pluralidad de visiones complementarias. En este nuevo ámbito de estudio, será necesario disponer del desarrollo de capacidades diferentes al abordar la realidad estudiada.

$\mathrm{Al}$ establecer un puente entre las diferentes ciencias naturales, las humanas y sociales, se hace posible una mayor comprensión del fenómeno de la vida, en otras palabras, se hace necesario acudir a la ciencia y la filosofía para comprender y hacer posible la vida y la cultura. En la postulación de esta nueva cultura o tercera cultura, como la llama Prigogine (1990), es indispensable un diálogo entre la experiencia conceptual y práctica de quienes intentan describir la sociedad humana en toda su complejidad y una modernización de la matemática; esto se traduce en una nueva cultura.

En relación con el tema de las disciplinas, se encuentra, además, que su estudio y la misión asignada a ellas desde la perspectiva de las ciencias de la complejidad parece agotarse; por ello, se hace necesario la formación interdisciplinaria, transdisciplinaria, multidisciplinaria, cruzada, transversal, es decir, integradora, ya no fragmentada y orientada hacia la especialización exclusivamente. Esta formación implica el reconocimiento de que los principales problemas a los que nos enfrentamos no se pueden resolver únicamente descomponiéndolos de forma analítica; es decir, las problemáticas que hacen referencia a la relación naturaleza-hombre-seres humanos deben ser abordadas de forma integral, holística y desde la complejidad de sus interacciones. Cada vez más se reconoce que las ciencias naturales han ido desplazándose hacia una visión del universo como inestable e impredecible, vislumbrándolo como una realidad activa e interdependiente con el hombre.

Además, las convergencias entre las ciencias sociales y las ciencias naturales se hacen cada día mayores en la medida en que ambas se interesan por el estudio de los sistemas complejos. Siguiendo a Wallerstein (2006), podemos establecer que: si bien la idea de utopía está íntimamente relacionada con la noción de progreso, el hecho de que se llegue a realizar no depende simplemente del avance de las ciencias naturales sino del desarrollo de la creatividad humana y de otro tipo de racionalidad para entender el mundo complejo que nos rodea:

\begin{abstract}
Venimos de un pasado social de certezas en conflicto, relacionadas con la ciencia, la ética o los sistemas sociales, a un presente de cuestionamiento considerable, incluyendo el cuestionamiento sobre la posibilidad intrínseca de la certeza. Es posible que estemos presenciando un tipo de racionalismo que no es apropiado para nuestro tiempo (p. 86).
\end{abstract}

\section{Conclusión}

Al estudiar el conocimiento dado por las ciencias naturales, sociales y humanas, se encuentra que existe pluralidad de métodos que de alguna manera llevan implícitas concepciones acerca de la sociedad y la cultura. Ello implica la necesidad de una reflexión sobre ellas, a la vez, una reflexión profunda de nuestro propio conocimiento y los supuestos sobre los que trabajamos.

Por otra parte, se advierte la inexistencia de fronteras nítidas entre la ciencia propiamente dicha y otras actividades del pensamiento humano. Ello plantea la necesidad de que el método científico tradicional abra o ensanche sus fronteras asumiendo una 
actitud de receptividad frente al aporte de la sociología, la historia de la ciencia, la lingüística, la dialéctica y la hermenéutica, la ciencia teológica, etc., con el fin de realizar una reconstrucción racional de las teorías científicas. Se hace imprescindible establecer canales de comunicación entre ciencia y sociedad.

Finalizando, se establece que: desde la inexistencia de fronteras nítidas y la riqueza producto de las intersecciones entre las diferentes disciplinas, se abona el terreno para el surgimiento de nuevos e innovadores problemas que antes eran considerados como resueltos o saldados. Mardones (1991) describe el panorama de las disciplinas en los siguientes términos:

Queda claro que no existen fronteras tan nítidas entre las ciencias y otras actividades del pensamiento humano. Crece, en suma, el convencimiento de la necesidad de profundizar la autoconciencia de la ciencia sobre su propio quehacer. La filosofía de la ciencia se vuelve reflexión sobre los presupuestos y supuestos de la tarea científica ( $\mathrm{p}$. 57).

\section{Parte}

Hombre y NATURALEZA DESDE LA VISIÓN DEL TAOÍSMO: APUNTES PARA UNA REFLEXIÓN SOBRE LA CRISIS ECOLÓGICOESPIRITUAL DEL HOMBRE ACTUAL

En toda morada resplandece la luz, somos nosotros los ciegos que no la vemos. Cuando a fuerza de mirar y remirar logremos percibirla finalmente, se rasgarán para nosotros los velos de este universo. -Kabir

En este apartado, se pretende hacer un breve recorrido por el tema de la relación hombre-naturaleza, desde la visión del taoísmo, milenaria tradición de la China antigua. Esta mirada sobre la complejidad de las relaciones entre el hombre y la naturaleza, desde la óptica del taoísmo, pretende establecer un diálogo con la manera como se ha abordado la problemática en el mundo occidental, sus posibles retos y aportes frente a un mundo cada vez más tecnocratizado y globalizado, signado por las desigualdades socio-económicas, las paradojas y contradicciones frente a sí mismo, a los demás y al universo.

\section{INTRODUCCIÓN}

Desde el cuestionamiento del llamado desarrollo humano en la época actual, que se ha distinguido por un constante incremento de la insolvencia racional del hombre y de su poder socio-económico para actuar con sabiduría y conciencia frente a la naturaleza y al cosmos, se hace necesario reflexionar so- 
bre éstas y muchas otras temáticas: el drama de la contaminación de la tierra, el cambio climático que se debe fundamentalmente a la acumulación de los gases de efecto invernadero, la difusión de las enfermedades infecciosas que dependen especialmente de las variables climáticas, la destrucción masiva de los bosques tropicales y los recursos no renovables de la tierra, las escasas políticas tendientes a frenar la creciente acumulación de desechos tóxicos de todo tipo, las aglomeraciones urbanas irracionales o el agotamiento de recursos básicos, como el agua potable, cada vez más escasa en grandes regiones de la tierra, el problema de la capa de ozono, y lo más preocupante, la zozobra de millones y millones de seres humanos condenados a vivir en condiciones de miseria y abandono.

Dichos fenómenos se presentan a la par que se determinan las posibilidades del desarrollo social y expansión económica de muchos bloques geopolíticos, que a su vez compiten por la obtención de los recursos naturales necesarios para la optimización de sus logros económicos, generando verdaderas batallas por obtener el liderazgo de los mercados locales, nacionales e internacionales, sin medir las consecuencias y el impacto que generan sobre el medio ambiente.

En contraste con lo anterior, el presente artículo analiza la relación que se estableció en las antiguas tradiciones de Oriente con respecto a la naturaleza. En dicha relación, podemos encontrar algunas claves para reorientar la posición del hombre contemporáneo frente a la naturaleza, en términos de unidad y de equilibrio correlacional.

\section{LAS RELACIONES HOMBRE- NATURALEZA EN LA ANTIGUA CHINA}

Perdí los límites de mi cuerpo físico. Sentí que estaba de pie en el centro del cosmos. Vi personas que venían hacia mí, pero eran todas el mismo hombre. Todas eran yo mismo. Nunca había visto este mundo. Yo había creído que estaba creado, pero ahora cambio de opinión: yo nunca fui creado, yo era el cosmos. Ninguno estaba separado de él. -Pensamiento Zen

Desde los escritos, leyendas y mitos expresados a través de las tradiciones de la antigua China, especialmente del taoísmo y del confucionismo, se evidencia un profundo espíritu de contemplación y relación mística con la naturaleza, además de una penetrante comprensión de su significado que se observa en las prácticas socio-culturales, políticas y místicas de dichas tradiciones y culturas; dichos hechos fundamentan un importante cuestionamiento y una reflexión en torno a las mismas relaciones y prácticas que hoy por hoy se establecen entre el hombre y la naturaleza.

En el caso de la tradición japonesa, el shintoismo o sintoísmo (shin: espíritu y to o do: camino) puede puntualizarse como una tradición naturalista, ya que en ella se venera a los espíritus y a los objetos de la naturaleza. Se considera que tanto los objetos como los fenómenos naturales tienen kami (o divinidad), es decir, poseen un carácter sagrado. Tratando de definirlos, se puede decir que los kami son seres extraordinarios, con saberes y capacidades superiores a los de los 
seres humanos, que habitan o se plasman en personas, objetos o seres naturales. Aunque su número es muy grande, se pueden sintetizar en tres grupos: los de las personas y sus antepasados, los de la naturaleza y sus fuerzas y los de los uji (o linajes). Algo interesante es que los kami más antiguos son los de la naturaleza, entre los cuales están los árboles, los pinos, las encinas y los especímenes más grandes. De la misma manera, también las rocas y las montañas, los ríos y lagos, también poseen kami. Cuentan las antiguas mitologías japonesas que los árboles hablaban y participaban de sus sabidurías a los seres humanos que se tomaban la molestia de oírlos.

Volviendo a la antigua China, según esta cultura, el tao originó el cielo y la tierra, como fuerza intermedia que regula el yinyang. Asimismo es el origen de todos los seres y el principio de todo dinamismo y movimiento. El tao es la ley natural del universo, por la cual cada persona puede llegar a superar el límite de la vida. Para alcanzar el tao, el ser humano debe tener paciencia, ser sencillo y sin pretensiones: debe practicar el principio del wu-wei (hacer sin esfuerzo): la persona superior siempre está en el wu wei, no actuando, sin pretender alcanzar un fin. La inferior siempre está haciendo lo que hay que hacer. El tao mismo siempre está en este estado, como si no hiciese nada, pero nada deja sin hacer. En el wu wei, se deja que las cosas sigan su curso natural, sin forzar las acciones de la naturaleza y del mismo hombre. Se considera que todo sucede según los designios del tao, además, en el mismo se desea la armonía entre la naturaleza y el cosmos entero.

Cuando el taoísta ha agregado el principio de wu-wei en su vida diaria, desarrolla una ma- nera innata de respuesta ante las demandas del mundo exterior: tanto desde su mismo cuerpo, como desde su mente, que actúa de manera espontánea, correcta, sin pensamiento, sin exigencias de lo que parece ser muy poco esfuerzo en la realización de un quehacer aparentemente difícil. Esta reacción natural del taoísta se fundamenta en un discernimiento de los principios, tiempos y ritmos que determinan el mismo universo, así como en la esencia del movimiento, que mora en la mente y el cuerpo humano. Wu wei no procede de la razón intelectual, sino de una especie de "inteligencia inconsciente" o innata en el ser humano, que sólo puede ser desarrollado a través del entrenamiento del tai chi, técnica marcial que concretiza esta filosofía o forma de vida taoísta. En palabras de Cooper (2001), al referirse al wu-wei, explica:

$$
\begin{aligned}
& \text { Se trata de la tranquila aceptación de la } \\
& \text { vida en el mundo tal como es y como } \\
& \text { viene; de aguardar el momento y la } \\
& \text { oportunidad, sin forzar nunca el resul- } \\
& \text { tado, sino que este se despliegue a su } \\
& \text { tiempo según su naturaleza (p. 34). }
\end{aligned}
$$

Lin Yu-tang, sabio taoísta, afirmaba que la gente divorciada de la naturaleza y que desconoce el sentido del wu wei tenía algunas características peculiares, entre ellas, que eran seres que se encontraban dominados por el propio ego, sólo les interesaba centrarse en sí mismos, se sentían solos y al desvío en un mundo adverso y se presentaban tácitamente o expresamente agresivo a sus vidas, o bien se angustiaban a sí mismos con emociones negativas de rivalidad, animadversión y rencor, o eran personas que juzgaban impetuosamente a la sociedad. Por último, según Yu-tang, estas personas no imaginaban la unidad última de todas las cosas y eran ciegas para ver que, al lastimar a la naturaleza, se lesionan a sí mismos. 
Por el contrario, quienes se encuentran en comunión con la naturaleza son conscientes de su verdadera naturaleza espiritual, crean en lugar de destruir, son consecuentes no sólo con su naturaleza humana sino que forman parte de una totalidad; esto les lleva necesariamente a un respeto profundo por toda la vida, una conciencia del significado de la existencia y del valor de toda experiencia o cosa; de esta manera, pueden aprender la sabiduría que se verifica en su originalidad y creatividad.

El sabio Ma Cheng Zhen (647-735 d. C) afirmaba:

El Tao es lo más importante para la vida y ésta hay que conciliarla siempre con aquél. La persona necesita el Tao, como el pez el agua. Si se defiende el Tao con la vida y con él se protege a ésta, se puede conseguir la longevidad (Ma Cheng Zhen, 2002, p. 23).

La misma palabra tao se utiliza como nombre general que se proporciona a la ley natural. La meta del tao es educar al ser humano para que se integre en la naturaleza, educándole en el fluir permanente con el cosmos, constituyéndose en sí mismo en una única realidad de armonía y equilibrio con el mismo. Propiamente el tao no es un creador porque nada en el mundo se crea, ni el mundo es creado; enseñar a compenetrarse con esta naturaleza y armonía de tal modo que llegue a experimentar en su propio cuerpo sus ritmos vitales, sintonizándose el cuerpo humano mediante una serie de ejercicios con estos ritmos, ganando así serenidad mental y salud física.

\section{El VACío TAOÍSTA COMO PRINCIPIO UNIFICADOR DEL CosMos}

Conserva el Vacío Absoluto

Y la perfecta Paz permanecerá

Todas las cosas tienen un mismo origen

$Y$ desde allí las contemplamos retornar

Todas las cosas emanan florecientes

$Y$ cada una de ellas regresa a su origen

Regresar a su Principio es reposar

Reposar es encontrar el nuevo Destino Al regreso al Destino se le llama Eternidad.

Al que conoce lo Eterno se le llama Iluminado

El que desconoce lo Eterno su miseria es desventura

Quien conoce la Eternidad todo lo posee

Quien es justo con los demás es soberano Quien es soberano es semejante a lo Supremo Lo Supremo es el Camino del Tao Alcanzando el Tao tendrá vida eterna Y aunque su cuerpo muera él nunca perecerá. -Lao Tse, Tao Te King

En el sentido occidental, el vacío equivale a carencia, a espacio por llenar, o se refiere a aquello que falta para que algo pueda considerarse o sentirse completo. De esta manera, puede designar tanto a una privación material, espiritual, emocional o al significado de la propia vida. En contraparte, se halla la proposición taoísta que eleva al vacío a un nivel primordial, como la sustancia de las cosas mismas y lo que, en definitiva, determina su sentido o propósito. Se destaca que el vacío taoísta es lo que no está, la ventana, el área libre, en un sentido concreto, que instituye, no obstante, un espacio dinámico y pleno de propósito, es decir, de contingencias y posibilidades de creación y de sentido. Como se concibe este concepto 
de vacío es aquel que otorga la cualidad a las formas visibles del universo.

De esta manera, en el taoísmo, este No-Ser o Vacío es igualmente el origen del Ser, y por medio del Ser, el principio de todas las cosas. En el texto sagrado del taoísmo, el Tao TeChing, se enuncia lo siguiente: "Todas las cosas bajo el Cielo son productos del Ser, pero el Ser mismo es el producto del No-Ser".

Desde esta profunda aseveración se percibe el origen de todo el pensamiento taoísta, al indicar la estructura profunda de la realidad y la subordinación de todo cuanto existe, respecto de aquello que es Infinito. Esto mismo se simboliza desde el concepto de vacío o no-ser ilimitado.

Chuang-Tzu, el más famoso filósofo de la antigua China, después de Lao Tsé, que vivió alrededor del siglo IV a. C., durante el período que corresponde a la cumbre del pensamiento filosófico chino, llamado "De las cien escuelas de pensamiento", considerado el segundo taoísta más importante, heredero del pensamiento de Lao Tsé, escribió, con respecto al tema del vacío:

En el Gran Principio (de todas las cosas) nada había en toda la vacatura del espacio; nada había que pudiera denominarse. Fue en este estado que nació la primera existencia -la primera existencia, pero aún sin forma corporal. De esta cosa pudo entonces producirse (recibiendo) lo que llamamos su propio carácter. Lo que no tenía forma corporal se dividió; y luego, sin intermedio, existió lo que llamamos el proceso de conferir. (Los dos procesos) siguieron operando, y se produjeron las líneas distintivas de cada una, que llamamos la forma corporal. Esa forma era el cuerpo que preserva en sí al espíritu, y cada uno tiene sus manifestaciones pe- culiares, que llamamos su Naturaleza. Cuando la Naturaleza fue cultivada, regresó a su propio carácter; y cuando se la alcanzó plenamente, se dio la misma condición que al comienzo (Legge, 1962, p. 45).

\section{EL TAO COMO MANIFESTACIÓN DEL PRINCIPIO Y DEL ORDEN}

Por último, es de capital importancia mencionar cómo el tao es el gran pedagogo en la cultura taoísta, al suministrar el camino para alcanzar el Principio y también el orden de las cosas; de hecho, es el orden de la naturaleza, al ser ley natural. Si se reflexiona todo lo que significa el tema del cuidado del universo, con respecto a la misma visión equilibrada de la naturaleza, se entiende la importancia de la relación hombre-cosmos, que subyace en el pensamiento taoísta: el tao, como Principio, es también el orden y la armonía de todas las cosas, está presente por doquier, está en todo lo que es grande o pequeño; así lo expresa el Tao-te-king (1983): “El Tao no se agota en lo que es máximo, ni está jamás ausente en lo que es mínimo; y por tanto se lo ha de hallar completo y difuso en todas las cosas" (p. 21).

Por esta razón, se enseña a los hombres, desde su niñez, a vivir en paz y armonía con el cielo y con la tierra, además de todos los seres que la pueblan; para alcanzar este principio pedagógico, el ser humano debe vivir de acuerdo con el tao y de conformidad con él, pues el tao impregna todas las cosas y también trasciende todas las cosas. La misma naturaleza, como el efecto directo del tao y sus leyes, se alza como contraria a las trivialidades de los artefactos humanos y a la artificialidad con la que el hombre se rodea. Pues, como lo dice el célebre maestro taoísta Chuang-zu (1987): 
Lo que es de la Naturaleza es interno. Lo que es del hombre es externo... El que bueyes y caballos deban tener cuatro patas es lo que pertenece a la Naturaleza. El que se deba poner un cabestro sobre la cabeza de un caballo, o una cuerda a través de la nariz del buey, es lo que pertenece al hombre. (p. 23)

Es por ello que el ideal educativo taoísta tiene como objetivo la formación del hombre espiritual, que es "contemplador" de la naturaleza: su meta es unificarse con ella, naturalizarse. Esto no se propone en sentido panteísta o naturalista, sino en sentido metafísico, de modo que volverse natural significa morar plenamente junto al tao que es, a la vez, trascendente y el principio de la naturaleza, pues a través de esta armonía llega la armonía con los hombres y esta armonía misma es el reflejo de la armonía con el cielo. Al respecto, escribe Chuang-Tzu lo siguiente:

Todo el que vea claramente la excelencia de toda la naturaleza puede llamarse Tronco de lo divino o Estirpe de lo divino, porque está en armonía con la naturaleza. Todo lo que ponga al mundo en acuerdo está en armonía con sus semejantes y feliz con los hombres. Quienquiera que esté en armonía con la naturaleza es feliz con la naturaleza (p. 11).

En síntesis, en las enseñanzas taoístas, ser feliz con la naturaleza significa precisamente aceptar sus normas y sus ritmos más que procurar someterla y sujetarla a los caprichos humanos. A la naturaleza no se la debe juzgar según la utilidad humana, ni el hombre debe convertirse soberbiamente en la medida de todas las cosas. No hay propiamente en la visión taoísta un antropomorfismo que vincule la relación del hombre con la naturaleza. Lo que debe hacer el hombre es aceptar y seguir a la naturaleza de las cosas y no tratar de alterar a la naturaleza por medios artificiales. La acción perfecta del ser humano es actuar sin autointerés y apego, o, en otras palabras, es actuar según la naturaleza que actúa libremente y sin codicia, sin motivos ulteriores o mezquinos. Se trata de una pedagogía que propone una reconexión del ser humano con los ritmos y las pulsaciones vitales del universo.

\section{CONCLUSIONES SOBRE EL APORTE DEL TAOÍSMO}

En este breve recorrido por la milenaria cultura China, especialmente desde el taoísmo, se puede evidenciar un ideario educativo y pedagógico, centrado en la armonía del ser humano con el cosmos, fundamentalmente con lo que denominamos "naturaleza". Desde el concepto del tao, como ley natural, que rige las pulsaciones del universo, se enseña al hombre a fluir con los ritmos del cosmos, respetando y armonizando su ser interior con las vibraciones del mundo externo, no ajeno e inherente a su condición energética. El taoísmo adquiere relevancia en nuestro tiempo porque ayuda a resolver la crisis ecológica creada por la visión judeocristiana que pretendió "dominar y someter la naturaleza". Es necesario recuperar el contacto con los ritmos de la naturaleza y con el fluir de las energías en el cuerpo. Lo que el mismo taoísmo denomina como la recuperación del rostro originario, la identidad perdida del universo. Pero su esencia está en el silencio, en el vacío, en el ritmo y en deseo de comunión con el cosmos.

En este momento crucial de la educación contemporánea, cuando se pregona con vehemencia propiciar una educación ambiental como proceso pedagógico dinámico y par- 
ticipativo que busca despertar en la población una conciencia que le permita identificarse con la problemática ambiental tanto a nivel colectivo como a nivel personal, los principios taoístas contribuyen a identificar las relaciones de interacción e independencia que se dan entre el entorno (mal llamado "medio ambiente") y el hombre, así como también contribuyen a propender por la promoción de una relación armónica entre el ser humano y la naturaleza mediante actividades ecológicas y antropogénicas, que hagan posible un desarrollo sostenible, garante del mantenimiento y la calidad de las generaciones actuales y futuras.

La pedagogía del taoísmo nos enseña a ubicar las causas de la fragmentación del hombre con su entorno natural, consigo mismo y con los demás, desde el análisis de la desconexión del mismo con el universo. De esta manera se deduce que toda propuesta educativa que tenga como fin la integridad total de la persona debe necesariamente establecer unos parámetros claros sobre la relación del mismo con su entorno natural y con el cosmos.

Lo anterior implica, asimismo, que la educación actual debe preocuparse por formar al ser humano para que asuma nuevos niveles de conciencia sobre su responsabilidad frente al drama de la destrucción del planeta tierra, además de apropiarse conscientemente de sus compromisos ético-ambientales.

\section{Conclusiones finales}

Aunque no es tarea fácil sacar conclusiones de las temáticas tratadas, se pueden, no obstante, apuntar las siguientes, en términos de nuevas preguntas, cuestionamientos y aperturas respecto a los enfoques científicos ac- tuales y a las tradiciones del lejano Oriente (en este caso, el taoísmo):

- Se destacan las limitaciones del pensamiento moderno y se propone una revisión y un aporte desde lo humano y desde las ciencias mismas, según la crítica realizada tanto desde los planteamientos filosóficos fenomenológicos de Husserl como desde las ciencias de la complejidad estudiadas por Prigogine.

- Con relación a la crítica emprendida desde las ciencias de la complejidad, se pronostica una metamorfosis en la orientación de las mismas ciencias. En esta crítica se muestra cómo el postulado determinista, que depende de forma categórica de la experimentación y se orienta fundamentalmente hacia la técnica, se torna cada vez más indiferente frente al mundo de la vida humana y la sociedad en general.

- Esta crítica va dirigida, además, y de forma especial, a la física clásica de corte positivista que pretendió constituirse como único conocimiento posible, descartando la validez de otros saberes posibles.

- Desde esta crítica se encuentra que la lógica presente en la ciencia moderna es necesariamente formal y, en su afán por destacar la legalidad universal $\mathrm{y}$ el determinismo, no tiene en cuenta el problema de la medición de las escalas (para la física de la modernidad: en cada instante, todo viene dado por el dato inicial con absoluta necesidad y predictibilidad), lo que equivale a afirmar que no existe diferencia alguna entre pasado $\mathrm{y}$ futuro (el tiempo reversible).

- Lo anterior trae como consecuencia el desconocimiento de singularidades, sobresaltos, accidentes e incertidumbres. 
Desde la física moderna (que trabaja el movimiento de los planetas o el péndulo ideal), existe un impedimento para plantear el tiempo asociado a la evolución biológica o el de las sociedades.

- La nueva posición epistemológica planteada desde las ciencias de la complejidad transformará radicalmente las relaciones del hombre con la naturaleza, los otros seres humanos y la sociedad en general. Desde Prigogine, la ciencia newtoniana no dialoga con la naturaleza sino que impone sus reglas fijas; además, el científico no solo no conoce la cosa en sí kantiana sino que también desconoce o no aborda los verdaderos problemas de la humanidad.

- La integridad fundamental hombrenaturaleza que pregona el taoísmo se puede plantear como fin primordial de algunos enfoques educativos, los cuales impulsan una nueva visión del hombre con respecto a la naturaleza. Esto implica una nueva concepción del desarrollo armónico del ser humano con el cosmos.

- Desde la cosmovisión taoísta, se percibe una invitación a la formación de "educadores ambientales", como estrategia que puede favorecer la educación ambiental de las presentes generaciones. Esta formación implica un trabajo interdiscipli- nario, derivado del carácter sistémico del ambiente y de la necesidad de aportar los instrumentos pedagógicos, de contenido y de acción desde las diversas disciplinas y áreas del conocimiento y las diversas perspectivas ecológicas contemporáneas que desean dar respuestas eficaces al drama ecológico de la tierra.

- Por último, el taoísmo como práctica pedagógica nos enseña que la capacidad de percepción no fragmentada en el hombre es el principio de la contemplación natural del universo. Dicho estado indiviso intensifica la sensibilidad y da lugar a la apertura del espacio y silencio interior, pilares de una nueva educación que busque los mejores métodos para solucionar los grandes problemas ambientales que se presentan en la actualidad, con el fin de frenar el deterioro ambiental al que hemos sometido a nuestro planeta tierra. Esta cualidad espiritual de integración hombre-cosmos es lo único que puede originar una nueva cultura en la que el conocimiento o saber de la ciencia tendría su justo lugar en el plano pedagógico y daría paso a un nuevo capítulo en el diálogo cienciaespiritualidad, desde el acercamiento a las tradiciones del lejano Oriente, particularmente, al taoísmo.

\section{REFERENCIAS}

Chuang Zu. (1983). Pensamientos del Maestro Chuang Tsé (I. Preciado Idoeta, Trad. y Ed.). Barcelona, España: Editorial Kairós.

Cooper, H. (2001). El Taoísmo. Barcelona, España: Editorial Base.
Córdoba, F. (1998). Fundamentos pedagógicos para la educación ambiental. Montería, Colombia: Fondo Editorial Universidad de Córdoba. 
Fung Yu-Lan. (1952). A history of Chinese philosophy (Vol. 1). New York, EE.UU.: Princeton University Press.

Guénon, R. (2004). La gran tríada. Barcelona, España: Editorial Paidós.

Herrera, D. (2002). La persona y el mundo de la experiencia. Bogotá, Colombia: Universidad San Buenaventura.

Husserl, E. (1965). Crítica a la ciencia moderna occidental. Milán, Italia: Editorial Il Saggiatore.

Husserl, E. (2008). La crisis de las ciencias europeas y la fenomenología trascendental. Buenos Aires, Argentina: Prometeo.

Iribarde, J. (2008). La crisis de las ciencias europeas y la fenomenología trascendental. Buenos Aires, Argentina: Prometeo.

Lao Zi. (1983). Tao Te Ching. Barcelona, España: Ediciones Teorema.

Lao Zi. (2006). Tao Te Ching: Los libros del Tao (I. Preciado Idoeta, Trad.). Madrid, España: Editorial Trotta.

Legge, L. (1962). The sacred books of China. The texts of taoism (Vol. 1). Nueva York, EE.UU.: New Books.

Lévi, J. (2005). Confucio (A. Galvany Larrouquere, Trad.). Madrid, España: Editorial Trotta.

Ma Cheng Zhen. (2002). Leyendas de China. En Sabiduría Oriental. Barcelona, España: Editorial Paidós.
Maldonado, E. (2005). Termodinámica y complejidad. Bogotá, Colombia: Universidad Externado de Colombia.

Ministerio de Educación Nacional de Colombia. (1998). Lineamientos curriculares en Ciencias Naturales y Educación Ambiental. Bogotá, Colombia: Editorial Libros \& Libros.

Morin, E. (1900). Introducción al pensamiento complejo. Barcelona, España: Gedisa.

Organización de las Naciones Unidas. (1972, junio). Declaración de la Conferencia de las Naciones Unidas sobre el Medio Ambiente Humano [Versión electrónica]. Disponible en http://www.dipublico. com.ar/ instrumentos/116.html

Prigogine, I. (1977). El fin de las certidumbres. Madrid, España: Taurus.

Prigogine, I. (1983). La nueva alianza. Madrid, España: Alianza Editorial.

Suárez, A. -H. (1997). Lunyu. Reflexiones y enseñanzas de Confucio. Barcelona, España: Clásicos Kairós.

Wallerstein, I. (2006). Abrir las ciencias sociales. Madrid, España: Siglo XXI.

Watts, A. (1962). El camino del Tao. California, EE.UU.: Celestial Arts Editions.

Wilheim, R. (Ed.). (2009). Tao Te King. Barcelona, España: Sirio.

Yu Dan. (2009). Confucio para el alma o las claves milenarias para ser feliz. Barcelona, España: Editorial Planeta. 